\title{
AN IDEAL FOR SELECTION OF SHORT-LIVED PARTICLES BY A FOCUSING CRYSTAL
}

\author{
Valery Biryukov* \\ IHEP Protvino, 142284 Moscow Region, Russia
}

\section{Abstract}

An experimental technique for selection of the short-lived particles by means of a focusing crystal is proposed. A target (to produce short-lived's) and a focusing crystal are to be arranged so that the crystal traps into channeling mode the particles emerging from the region downstream of the target only. The particles emerging from the target directly, cannot be trapped in the crystal. In this way one can trap the decay products of short-lived particles to carry them out of the background; the trapped beam can be bent, e.g., onto the experimental set up. The technique can handle the particles with decay length $c \tau$ down to few micron. The capabilities of the technique, resolution, and efficiency are analyzed.

\section{INTRODUCTION}

Short-lived particles such as B-mesons have the lifetimes $\tau$ so short that $c \tau$ equals to only $\sim 100-400 \mu \mathrm{m}$. Even with relativistic factor of $\sim 100$ the mean path before decay for these particles equals only $\sim 1-4 \mathrm{~cm}$. There is no way to handle these particles by a traditional magnet beam optics. The bent-crystal-channeling technique allows to steer the particle beams, bend or focus them, along a few $\mathrm{cm}$ path, with equivalent fields on the order of 1000 Tesla and more [1]. Applications of this technique to short-lived particles have been proposed [2,3]. One application-a measurement of the particle's magnetic moment in a bent crystalwas successfully tested in the experiment [4].

\section{THE PRINCIPLE}

The discussed idea involves a focusing crystal, which can either focus a parallel beam into a point, or perform a reversed process, i.e. trap a beam emerging from a point-like source; both applications have been demonstrated experimentally [1]. Suppose, a target (to produce short-lived's) and a focusing crystal are arranged so that the crystal focal point $\mathrm{F}$ is outside of the target (Fig. 1). Only the particles emerging from $\mathrm{F}$ can be trapped by the crystal. Then, if some particle decays at $\mathrm{F}$, all the decay products will be trapped. If some particle emerges from the target directly, it is not trapped, unless it passes through F; this consideration sets a "blind spot" B on the crystal face, where one cannot distinguish between the particles of interest and the garbage. The trapped particles can be bent, e.g. onto some experimental set up (bending efficiencies up to $60 \%$ have recently been demonstrated [5]).

\footnotetext{
*E-mail: biryukov@mx.ihep.su
}

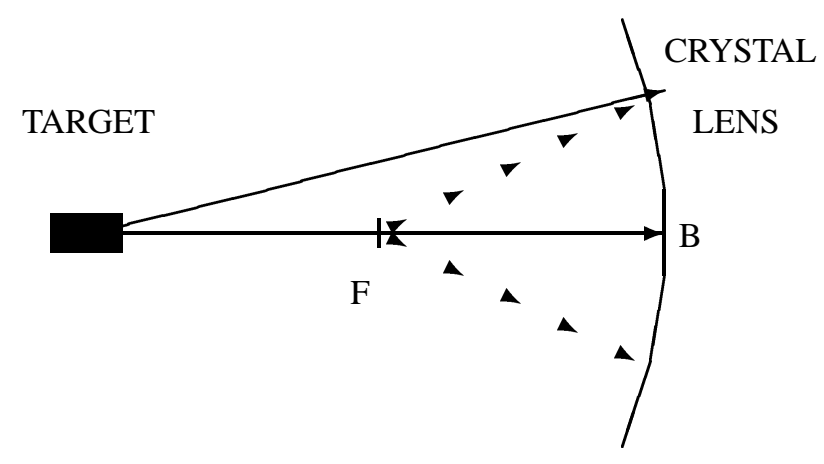

Figure 1: Schematic arrangement of a target, focusing crystal, and the focus F. "Blind spot" is also shown (B).

With the principle understood, let's understand the limitations. Crystal traps a particle if it comes within a Lindhard angle $\theta_{L}$, which is e.g. $5 \mu \mathrm{rad} / E^{1 / 2}(\mathrm{TeV})$ in $\mathrm{Si}(110)$ planes at energy $E$. Therefore, the focus is not a precise point, but has a transverse size of $\pm \theta_{L} L$, where the focal length $L$ is as set by the crystal design (e.g. it was $0.5-4 \mathrm{~m}$ in the experiment[1]); in Fig. 1, $L$ is the distance between points $\mathrm{F}$ and $\mathrm{B}$.

The typical angle $\theta_{D}$ under which the decay products emerge from $\mathrm{F}$, is set by the relativistic factor $\gamma$ of the parent particle: $\theta_{D} \simeq 1 / \gamma$. Therefore, the longitudinal size of the focal spot equals $\theta_{L} L / \theta_{D} \simeq \gamma \theta_{L} L$. Only the particles emerging from this focal spot of the length $\gamma \theta_{L} L$ and of the width $\theta_{L} L$ can be trapped by the crystal (Fig. 2).

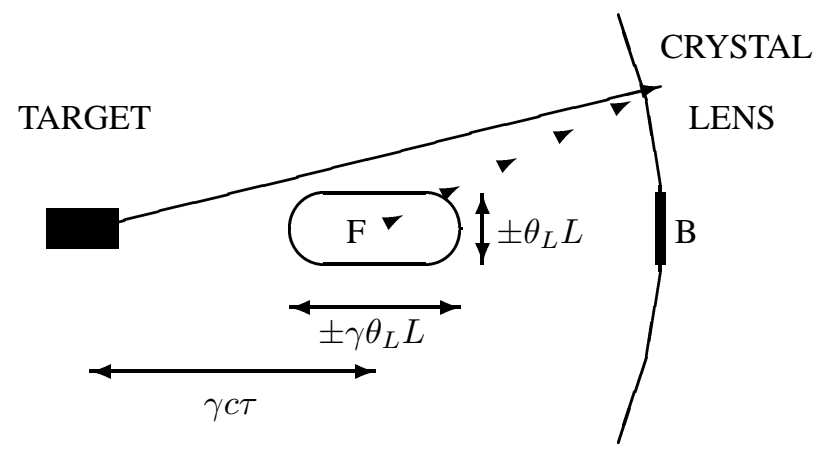

Figure 2: Schematic of a target and the focal spot dimenions. The "blind spot" is shown (B).

A particle with relativistic factor $\gamma$ and the lifetime $\tau$ has 
a mean decay path of $\gamma c \tau$. The discussed scheme makes sense only if $\gamma c \tau$ is on the order of or larger than $\gamma \theta_{L} L$. Hence, our technique can handle particles with $c \tau$ as small as $\theta_{L} L$. For the short-lived particles (with beauty or charm, or $\tau$-leptons etc.) $c \tau$ varies from 50 to $400 \mu \mathrm{m}$. The minimal $\theta_{L} L$ already obtained in the experiment at $70 \mathrm{GeV}$ was $40 \mu \mathrm{m} \mathrm{[1].} \mathrm{At} \mathrm{higher} \mathrm{energy} E$ the technique resolution $\theta_{L} L$ improves like $E^{-1 / 2}$. Moreover, $L$ is defined by just practical convenience. Again, $\theta_{L}$ can be chosen smaller og bigger from different crystal planes or axes. E.g., using $L=0.2 \mathrm{~m}$ at $500 \mathrm{GeV}$ gives the minimal resolution of $\sim 1$ $\mu \mathrm{m}$. If one doesn't care on fine resolution for $c \tau$ but wish to increase the focal spot just to increase the working space, this is easy to do by means of greater $L$.

The angular acceptance of the crystal should be about $\pm 1 / \gamma$. The "blind spot" angular range in the crystal center is set by the focal spot transverse size $\theta_{L} L$ and the targetto-focus distance, about $\gamma c \tau$. The "blind" range is then $\pm \theta_{L} L / \gamma c \tau$. Its ratio to the crystal angular acceptance, i.e. the crystal inefficiency, equals $\theta_{L} L / c \tau$. As from above, this inefficiency is on the order of $1 \%$.

We can also estimate the rejection factor for the particles incident beyond the Lindhard angle. They may be trapped in crystal through the scattering processes only, so called feed-in or volume capture. The probability of feed-in as measured at $70 \mathrm{GeV}$ is on the order of $10^{-3}$. Therefore only $\sim 10^{-3}$ of the background is not rejected by the crystal and hence mixed up with the "particles of interest".

Unfortunately, a high efficiency trapping and bending has been demonstrated so far for positive particles only. Therefore, a major drawback of the discussed scheme (as well as of any other application of bent crystals) is its restriction to only positive particles produced in a decay.

\section{CONCLUSION}

The proposed technique can trap the decay products of the short-lived particles and bend them toward an experimental set-up. The rejection factor for the particles produced in the target is $\sim 1000$. The technique can handle particles with $c \tau$ down to $\sim 1 \mu \mathrm{m}$.

\section{REFERENCES}

[1] V.Biryukov, Yu.Chesnokov, V.Kotov. Crystal Channeling and its Application at High Energy Accelerators. (Springer, Berlin: 1997)

[2] R.A.Carrigan, Jr. in Relativistic Channeling. R.A.Carrigan, Jr. and J.Ellison, eds. (Plenum, NY, 1987) p. 339

[3] C.R.Sun in Relativistic Channeling. R.A.Carrigan, Jr. and J.Ellison, eds. (Plenum, NY, 1987) p.379

[4] D.Chen et al. Phys Rev. Lett, 69, 3286 (1992)

[5] C.Biino et al., to appear in Phys.Lett. B 